APPLICATIONES MATHEMATICAE

26,4 (1999), pp. 415-435

W. HAN (Iowa City) and M. SOFONEA (Perpignan)

\title{
ANALYSIS AND NUMERICAL APPROXIMATION OF AN ELASTIC FRICTIONAL CONTACT PROBLEM WITH NORMAL COMPLIANCE
}

Abstract. We consider the problem of frictional contact between an elastic body and an obstacle. The elastic constitutive law is assumed to be nonlinear. The contact is modeled with normal compliance and the associated version of Coulomb's law of dry friction. We present two alternative yet equivalent weak formulations of the problem, and establish existence and uniqueness results for both formulations using arguments of elliptic variational inequalities and fixed point theory. Moreover, we show the continuous dependence of the solution on the contact conditions. We also study the finite element approximations of the problem and derive error estimates. Finally, we introduce an iterative method to solve the resulting finite element system.

1. Introduction. Processes of frictional contact between deformable bodies are very common in industry and everyday life. Contact without lubrication can be found for example in the process of metal forming, in car's braking systems, in engines, motors and transmissions. Despite the difficulties that the process of frictional contact presents because of the complicated surface phenomena involved, considerable progress has been made in the modeling and analysis of contact problems. An early attempt to study frictional contact problems for elastic and viscoelastic materials within the framework of variational inequalities was made in [6]. Steadystate as well as time-dependent frictional contact problems for linearly and nonlinearly elastic materials may be found in [13]. An excellent reference to the field of contact problems with or without friction is [9].

1991 Mathematics Subject Classification: 73C35, 73T05, 35J85, 73V05.

Key words and phrases: frictional contact, normal compliance, Coulomb's law, variational inequality, fixed point, finite element approximation, error estimates. 
Dynamic problems with normal compliance contact condition were first considered in [11] where the existence of a weak solution was proven. This condition allows the interpenetration of the body's surface into the obstacle. It was justified in [11] and [10] by considering the interpenetration and deformation of surface asperities. Moreover, the normal compliance condition has been employed as a mathematical regularization of Signorini's nonpenetration condition and used in numerical solution algorithms. Contact problems with normal compliance have been discussed in numerous papers, e.g. [1, 7 , $9,10,14]$ and the references therein.

In this work we consider the problem of frictional contact between an elastic body and a foundation. We assume that the forces and tractions acting upon the body change slowly in time so that the acceleration in the system is negligible. Neglecting the inertial term in the equation of motion leads to a quasistatic approximation for the process. The material's constitutive law is assumed to be nonlinear. The contact is modeled with a normal compliance condition and the static version of Coulomb's law of dry friction. We establish the existence of a unique solution to the problem, using fixed point arguments. Then we prove the stability of the solution with respect to perturbation of the normal compliance functions, which is important from the point of view of applications. We also discuss the numerical treatment of the problem and derive some error estimates.

The paper is organized as follows. In Section 2 we introduce the function spaces for various quantities, and state assumptions on given data. The mechanical problem is stated in Section 3, where two alternative yet equivalent variational forms of the problem are formulated. The unknown for one of the variational problems is the displacement, while for the other, the unknown is the stress. For both variational problems, we show the unique solvability in Section 4. The proofs are based on arguments from elliptic variational inequalities and fixed point properties of certain maps. We also study the link between the solutions of the variational problems and we prove that the displacement and the stress field are related by the elastic constitutive law. Section 5 is devoted to a result on the continuous dependence of the solution on contact conditions, which indicates that a small perturbation in the contact condition leads to a small change in the solution. Finally, in Section 6 we study the finite element approximation of the displacement variational formulation. We prove Céa's type inequalities, from which we can conclude the convergence of the finite element method and derive order error estimates under appropriate regularity assumptions on the exact solution. We introduce an iterative method to solve the resulting finite element system, which converges under certain assumptions. 
2. Preliminaries. Let us introduce various notations and spaces which will be used in the formulation and analysis of the mechanical problem. For further details on this preliminary material we refer the reader to $[6,8,13]$.

Let $\Omega$ be a bounded domain in $\mathbb{R}^{N}(N=2,3$ in applications) with a Lipschitz boundary $\Gamma$ and let $\Gamma_{1}$ be a measurable part of $\Gamma$ such that meas $\left(\Gamma_{1}\right)>0$. Since the boundary is Lipschitz continuous, the unit outward normal vector $\boldsymbol{\nu}$ is defined a.e. on $\Gamma$.

Let $S_{N}$ represent the space of second order symmetric tensors on $\mathbb{R}^{N}$, or equivalently, the space of symmetric matrices of order $N$. We define the inner products and the corresponding norms on $\mathbb{R}^{N}$ and $S_{N}$ by

$$
\begin{aligned}
& \boldsymbol{u} \cdot \boldsymbol{v}=u_{i} v_{i}, \quad|\boldsymbol{v}|=(\boldsymbol{v} \cdot \boldsymbol{v})^{1 / 2}, \quad \forall \boldsymbol{u}, \boldsymbol{v} \in \mathbb{R}^{N}, \\
& \boldsymbol{\sigma} \cdot \boldsymbol{\tau}=\sigma_{i j} \tau_{i j}, \quad|\boldsymbol{\tau}|=(\boldsymbol{\tau} \cdot \boldsymbol{\tau})^{1 / 2}, \quad \forall \boldsymbol{\sigma}, \boldsymbol{\tau} \in S_{N} .
\end{aligned}
$$

Here and below, $i, j=1, \ldots, N$, and the summation convention over repeated indices is adopted. Moreover, in the sequel, the index that follows a comma indicates a partial derivative, e.g., $u_{i, j}=\partial u_{i} / \partial x_{j}$.

We now introduce several function spaces. Let

$$
\begin{array}{ll}
H=\left\{\boldsymbol{u}=\left(u_{i}\right) \mid u_{i} \in L^{2}(\Omega)\right\}, & H_{1}=\left\{\boldsymbol{u}=\left(u_{i}\right) \mid u_{i} \in H^{1}(\Omega)\right\}, \\
\mathcal{H}=\left\{\boldsymbol{\sigma}=\left(\sigma_{i j}\right) \mid \sigma_{i j}=\sigma_{j i} \in L^{2}(\Omega)\right\}, & \mathcal{H}_{1}=\left\{\boldsymbol{\sigma} \in \mathcal{H} \mid \sigma_{i j, j} \in H\right\} .
\end{array}
$$

The spaces $H, \mathcal{H}, H_{1}$ and $\mathcal{H}_{1}$ are real Hilbert spaces endowed with the inner products given by

$$
\begin{aligned}
(\boldsymbol{u}, \boldsymbol{v})_{H} & =\int_{\Omega} u_{i} v_{i} d x, \quad(\boldsymbol{u}, \boldsymbol{v})_{H_{1}}=(\boldsymbol{u}, \boldsymbol{v})_{H}+(\boldsymbol{\varepsilon}(\boldsymbol{u}), \boldsymbol{\varepsilon}(\boldsymbol{v}))_{\mathcal{H}}, \\
(\boldsymbol{\sigma}, \boldsymbol{\tau})_{\mathcal{H}} & =\int_{\Omega} \sigma_{i j} \tau_{i j} d x, \quad(\boldsymbol{\sigma}, \boldsymbol{\tau})_{\mathcal{H}_{1}}=(\boldsymbol{\sigma}, \boldsymbol{\tau})_{\mathcal{H}}+(\operatorname{Div} \boldsymbol{\sigma}, \operatorname{Div} \boldsymbol{\tau})_{H} .
\end{aligned}
$$

Here $\varepsilon: H_{1} \rightarrow \mathcal{H}$ and Div $: \mathcal{H}_{1} \rightarrow H$ are the deformation and the divergence operators, defined by

$$
\boldsymbol{\varepsilon}(\boldsymbol{u})=\left(\varepsilon_{i j}(\boldsymbol{u})\right), \quad \varepsilon_{i j}(\boldsymbol{u})=\frac{1}{2}\left(u_{i, j}+u_{j, i}\right), \quad \operatorname{Div} \boldsymbol{\sigma}=\left(\sigma_{i j, j}\right) .
$$

The associated norms are denoted by $\|\cdot\|_{H},\|\cdot\|_{\mathcal{H}},\|\cdot\|_{H_{1}}$ and $\|\cdot\|_{\mathcal{H}_{1}}$.

Let $H_{\Gamma}=H^{1 / 2}(\Gamma)^{N}$ and let $\gamma: H_{1} \rightarrow H_{\Gamma}$ be the trace map. For every element $\boldsymbol{v} \in H_{1}$, we also use the notation $\boldsymbol{v}$ to denote the trace $\gamma \boldsymbol{v}$ of $\boldsymbol{v}$ on $\Gamma$ and we denote by $v_{\nu}$ and $\boldsymbol{v}_{\tau}$ the normal and tangential components of $\boldsymbol{v}$ on $\Gamma$ given by

$$
v_{\nu}=\boldsymbol{v} \cdot \boldsymbol{\nu}, \quad \boldsymbol{v}_{\tau}=\boldsymbol{v}-v_{\nu} \boldsymbol{\nu}
$$

Let $H_{\Gamma}^{\prime}$ be the dual of $H_{\Gamma}$ and let $\langle\cdot, \cdot\rangle$ denote the duality pairing between $H_{\Gamma}^{\prime}$ and $H_{\Gamma}$. For every $\boldsymbol{\sigma} \in \mathcal{H}_{1}, \boldsymbol{\sigma} \boldsymbol{\nu}$ can be defined as the element in $H_{\Gamma}^{\prime}$ which satisfies

$$
\langle\boldsymbol{\sigma} \boldsymbol{\nu}, \gamma \boldsymbol{v}\rangle=(\boldsymbol{\sigma}, \boldsymbol{\varepsilon}(\boldsymbol{v}))_{\mathcal{H}}+(\operatorname{Div} \boldsymbol{\sigma}, \boldsymbol{v})_{H} \quad \forall \boldsymbol{v} \in H_{1} .
$$


Denote by $\sigma_{\nu}$ and $\boldsymbol{\sigma}_{\tau}$ the normal and tangential traces of $\boldsymbol{\sigma}$, respectively. If $\boldsymbol{\sigma}$ is continuously differentiable on $\bar{\Omega}$, then

$$
\begin{gathered}
\sigma_{\nu}=(\boldsymbol{\sigma} \boldsymbol{\nu}) \cdot \boldsymbol{\nu}, \quad \boldsymbol{\sigma}_{\tau}=\boldsymbol{\sigma} \boldsymbol{\nu}-\sigma_{\nu} \boldsymbol{\nu} \\
\langle\boldsymbol{\sigma} \boldsymbol{\nu}, \gamma \boldsymbol{v}\rangle=\int_{\Gamma} \boldsymbol{\sigma} \boldsymbol{\nu} \cdot \boldsymbol{v} d a
\end{gathered}
$$

for all $\boldsymbol{v} \in H_{1}$, where $d a$ is the surface measure element.

In the sequel we use $V$ to denote the closed subspace of $H_{1}$ defined by

$$
V=\left\{\boldsymbol{v} \in H_{1} \mid \boldsymbol{v}=\mathbf{0} \text { on } \Gamma_{1}\right\} .
$$

Since meas $\left(\Gamma_{1}\right)>0$, the following Korn inequality holds:

$$
\|\varepsilon(\boldsymbol{v})\|_{\mathcal{H}} \geq c_{K}\|\boldsymbol{v}\|_{H_{1}} \quad \forall \boldsymbol{v} \in V
$$

(see e.g. [12]). Here $c_{K}$ denotes a strictly positive constant which depends only on $\Omega$ and $\Gamma_{1}$.

On $V$ we consider the inner product given by

$$
(\boldsymbol{u}, \boldsymbol{v})_{V}=(\varepsilon(\boldsymbol{u}), \boldsymbol{\varepsilon}(\boldsymbol{v}))_{\mathcal{H}}
$$

and let $\|\cdot\|_{V}$ be the associated norm. It follows from Korn's inequality (2.5) that $\|\cdot\|_{H_{1}}$ and $\|\cdot\|_{V}$ are equivalent norms on $V$. Therefore $\left(V,\|\cdot\|_{V}\right)$ is a real Hilbert space.

3. The mechanical problem and weak formulations. In this section we introduce the physical setting, list the assumptions on the problem data and present the variational formulations of the model.

We consider an elastic body occupying a bounded domain $\Omega \subset \mathbb{R}^{N}$ with a Lipschitz boundary $\Gamma$, partitioned into three disjoint measurable parts $\Gamma_{1}$, $\Gamma_{2}$ and $\Gamma_{3}$ such that meas $\left(\Gamma_{1}\right)>0$. A volume force of density $\varphi_{1}$ acts in $\Omega$ and a surface traction of density $\varphi_{2}$ acts on $\Gamma_{2}$. The body is clamped on $\Gamma_{1}$ and thus the displacement field vanishes there. A gap $g$ exists between the potential contact surface $\Gamma_{3}$ and a foundation, and it is measured along the direction of the outward normal $\boldsymbol{\nu}$.

We denote by $\boldsymbol{u}$ the displacement vector, $\boldsymbol{\sigma}$ the stress field and $\boldsymbol{\varepsilon}(\boldsymbol{u})$ the small strain tensor. The elastic constitutive law of the material is assumed to be

$$
\boldsymbol{\sigma}=F(\varepsilon(\boldsymbol{u}))
$$

with a given (nonlinear) function $F$. Here and below, in order to simplify the notation, we usually do not indicate explicitly the dependence of various functions on the spatial variable $\boldsymbol{x} \in \Omega \cup \Gamma$.

Next, we describe the condition on the potential contact surface $\Gamma_{3}$. We assume that the normal stress $\sigma_{\nu}$ satisfies the normal compliance condition

$$
-\sigma_{\nu}=p_{\nu}\left(u_{\nu}-g\right)
$$


where $u_{\nu}$ represents the normal displacement, $p_{\nu}$ is a prescribed nonnegative function with $p_{\nu}(t)=0$ for $t \leq 0$, and $u_{\nu}-g$, when it is positive, represents the penetration of the body in the foundation. Such a contact condition was proposed in [11] and used in a number of publications (see e.g. [1, 7, $9,10,14]$ and references therein). In this condition the interpenetration is allowed but penalized. In $[7,10,11]$ the following function was employed:

$$
p_{\nu}(t)=c_{\nu}(t)_{+}^{m_{\nu}}
$$

where $c_{\nu}$ is a positive constant, $m_{\nu}$ is a positive exponent and $t_{+}=\max \{0, t\}$. Formally, Signorini's nonpenetration condition is obtained in the limit $c_{\nu} \rightarrow$ $\infty$. Here we allow for a more general expression, similar to the one used in $[1,14]$.

The associated friction law on $\Gamma_{3}$ is chosen as

$$
\left\{\begin{array}{l}
\left|\boldsymbol{\sigma}_{\tau}\right| \leq p_{\tau}\left(u_{\nu}-g\right), \\
\left|\boldsymbol{\sigma}_{\tau}\right|<p_{\tau}\left(u_{\nu}-g\right) \Rightarrow \boldsymbol{u}_{\tau}=\mathbf{0}, \\
\left|\boldsymbol{\sigma}_{\tau}\right|=p_{\tau}\left(u_{\nu}-g\right) \Rightarrow \boldsymbol{\sigma}_{\tau}=-\lambda \boldsymbol{u}_{\tau}, \quad \lambda \geq 0
\end{array}\right.
$$

Here $p_{\tau}$ is a nonnegative function, the so-called friction bound, which satisfies $p_{\tau}(t)=0$ for $t \leq 0, \boldsymbol{u}_{\tau}$ denotes the tangential displacement and $\boldsymbol{\sigma}_{\tau}$ represents the tangential force on the contact boundary. This is a static version of Coulomb's law of dry friction and should be seen either as a mechanical model suitable for the proportional loadings or as a first approximation of a more realistic model, based on a friction law involving the time derivative of $\boldsymbol{u}_{\tau}$ (see for instance $[14,15]$ ). It states that the tangential shear cannot exceed the maximal frictional resistance $p_{\tau}$. When strict inequality holds the surface adheres to the foundation and is in the so-called stick state, and when equality holds there is relative sliding, the so-called slip state. Therefore, the contact surface $\Gamma_{3}$ is divided into three zones: stick, slip and the separation zone in which $u_{\nu}<g$, i.e. there is no contact. The boundaries of these zones are free boundaries since they are unknown a priori, and are part of the problem.

In the references $[3,4,5,6,9]$, the friction law (3.4) was used with

$$
p_{\tau}=\mu p_{\nu}
$$

where $\mu>0$ is a coefficient of friction. In [7] the friction law (3.4) was used with

$$
p_{\tau}(t)=c_{\tau}
$$

where $c_{\tau}>0$. Recently, a new version for Coulomb's law of friction was derived in $[16,17]$ from thermodynamic consideration. It consists in using in (3.4) the friction bound function

$$
p_{\tau}=\mu p_{\nu}\left(1-\alpha p_{\nu}\right)_{+}
$$


where $\alpha$ is a small positive coefficient related to the wear and hardness of the surface.

With (3.1) as the constitutive relation, (3.2) and (3.4) for the contact condition, the mechanical problem of frictional contact of the elastic body may be formulated classically as follows.

Problem $P$. Find a displacement field $\boldsymbol{u}: \Omega \rightarrow \mathbb{R}^{N}$ and a stress field $\sigma: \Omega \rightarrow S_{N}$ such that

$$
\begin{array}{ll}
\boldsymbol{\sigma}=F(\boldsymbol{\varepsilon}(\boldsymbol{u})) & \text { in } \Omega, \\
\operatorname{Div} \boldsymbol{\sigma}+\boldsymbol{\varphi}_{1}=\mathbf{0} & \text { in } \Omega, \\
\boldsymbol{u}=\mathbf{0} & \text { on } \Gamma_{1}, \\
\boldsymbol{\sigma} \boldsymbol{\nu}=\boldsymbol{\varphi}_{2} & \text { on } \Gamma_{2},
\end{array}
$$

and on $\Gamma_{3}$,

$$
\left\{\begin{array}{l}
-\sigma_{\nu}=p_{\nu}\left(u_{\nu}-g\right), \\
\left|\boldsymbol{\sigma}_{\tau}\right| \leq p_{\tau}\left(u_{\nu}-g\right), \\
\left|\boldsymbol{\sigma}_{\tau}\right|<p_{\tau}\left(u_{\nu}-g\right) \Rightarrow \boldsymbol{u}_{\tau}=\mathbf{0}, \\
\left|\boldsymbol{\sigma}_{\tau}\right|=p_{\tau}\left(u_{\nu}-g\right) \Rightarrow \boldsymbol{\sigma}_{\tau}=-\lambda \boldsymbol{u}_{\tau} \text { for some } \lambda \geq 0 .
\end{array}\right.
$$

In the study of the mechanical problem (3.8)-(3.12) we assume that the elasticity operator

$$
F: \Omega \times S_{N} \rightarrow S_{N}
$$

satisfies

(3.13) (a) There exists an $M>0$ such that $\left|F\left(\boldsymbol{x}, \boldsymbol{\varepsilon}_{1}\right)-F\left(\boldsymbol{x}, \boldsymbol{\varepsilon}_{2}\right)\right| \leq M\left|\varepsilon_{1}-\varepsilon_{2}\right|$ for all $\varepsilon_{1}, \varepsilon_{2} \in S_{N}$, a.e. in $\Omega$.

(b) There exists an $m>0$ such that $\left.\left(F\left(\boldsymbol{x}, \varepsilon_{1}\right)\right)-F\left(\boldsymbol{x}, \boldsymbol{\varepsilon}_{2}\right)\right) \cdot\left(\varepsilon_{1}-\varepsilon_{2}\right)$ $\geq m\left|\varepsilon_{1}-\varepsilon_{2}\right|^{2}$ for all $\varepsilon_{1}, \varepsilon_{2} \in S_{N}$, a.e. in $\Omega$.

(c) The mapping $\boldsymbol{x} \mapsto F(\boldsymbol{x}, \boldsymbol{\varepsilon})$ is Lebesgue measurable on $\Omega$ for any $\varepsilon \in S_{N}$.

(d) $F(\boldsymbol{x}, \mathbf{0}) \in \mathcal{H}$ for all $\boldsymbol{x} \in \Omega$.

A family of elasticity operators satisfying the condition (3.13) is provided by nonlinear Hencky materials (for details, cf. e.g. [18]). For a Hencky material, the stress-strain relation is

$$
\boldsymbol{\sigma}=K_{0} \operatorname{tr} \boldsymbol{\varepsilon}(\boldsymbol{u}) \boldsymbol{I}+\psi\left(\left|\varepsilon^{D}(\boldsymbol{u})\right|^{2}\right) \varepsilon^{D}(\boldsymbol{u}),
$$

so that the elasticity operator is

$$
F(\varepsilon)=K_{0} \operatorname{tr} \varepsilon \boldsymbol{I}+\psi\left(\left|\varepsilon^{D}\right|^{2}\right) \varepsilon^{D} .
$$

Here, $K_{0}>0$ is a material coefficient, $\boldsymbol{I}$ is the identity tensor of the second order, $\operatorname{tr} \varepsilon=\varepsilon_{i i}$ is the trace of $\varepsilon$, and $\varepsilon^{D}$ denotes the deviatoric part of $\varepsilon$ :

$$
\varepsilon^{D}=\varepsilon-\frac{1}{N} \operatorname{tr} \varepsilon \boldsymbol{I}
$$


The function $\psi$ is assumed to be piecewise continuously differentiable, and there exist positive constants $c_{1}, c_{2}, d_{1}$ and $d_{2}$ such that for $\xi \geq 0$,

$$
\psi(\xi) \leq d_{1}, \quad-c_{1} \leq \psi^{\prime}(\xi) \leq 0, \quad c_{2} \leq \psi(\xi)+2 \psi^{\prime}(\xi) \xi \leq d_{2} .
$$

Let us show that the condition (3.13) is satisfied for the elasticity operator defined in (3.14). We have

$$
\begin{aligned}
F\left(\varepsilon_{1}\right)- & F\left(\varepsilon_{2}\right) \\
= & K_{0} \operatorname{tr}\left(\varepsilon_{1}-\varepsilon_{2}\right) \boldsymbol{I}+\psi\left(\left|\varepsilon_{1}^{D}\right|^{2}\right) \varepsilon_{1}^{D}-\psi\left(\left|\varepsilon_{2}^{D}\right|^{2}\right) \varepsilon_{2}^{D} \\
= & K_{0} \operatorname{tr}\left(\varepsilon_{1}-\varepsilon_{2}\right) \boldsymbol{I}+\int_{0}^{1} \frac{d}{d t}\left[\psi\left(\left|\varepsilon_{2}^{D}+t\left(\varepsilon_{1}^{D}-\varepsilon_{2}^{D}\right)\right|^{2}\right)\left(\varepsilon_{2}^{D}+t\left(\varepsilon_{1}^{D}-\varepsilon_{2}^{D}\right)\right)\right] d t \\
= & K_{0} \operatorname{tr}\left(\varepsilon_{1}-\varepsilon_{2}\right) \boldsymbol{I} \\
& +\int_{0}^{1}\left[2 \psi^{\prime}\left(\left|\varepsilon_{2}^{D}+t\left(\varepsilon_{1}^{D}-\varepsilon_{2}^{D}\right)\right|^{2}\right)\right. \\
& \times\left(\varepsilon_{2}^{D}+t\left(\varepsilon_{1}^{D}-\varepsilon_{2}^{D}\right)\right) \cdot\left(\varepsilon_{1}-\varepsilon_{2}\right)\left(\varepsilon_{2}^{D}+t\left(\varepsilon_{1}^{D}-\varepsilon_{2}^{D}\right)\right) \\
& \left.+\psi\left(\left|\varepsilon_{2}^{D}+t\left(\varepsilon_{1}^{D}-\varepsilon_{2}^{D}\right)\right|^{2}\right)\left(\varepsilon_{1}-\varepsilon_{2}\right)\right] d t .
\end{aligned}
$$

Then the condition (3.13)(a) is satisfied for some constant $M$ depending on $K_{0}, d_{1}, d_{2}$ and $c_{1}$. Now

$$
\begin{aligned}
\left(F\left(\varepsilon_{1}\right)-\right. & \left.F\left(\varepsilon_{2}\right)\right) \cdot\left(\varepsilon_{1}-\varepsilon_{2}\right) \\
= & K_{0}\left|\operatorname{tr}\left(\varepsilon_{1}-\varepsilon_{2}\right)\right|^{2} \\
& \quad+\int_{0}^{1}\left[2 \psi^{\prime}\left(\left|\varepsilon_{2}^{D}+t\left(\varepsilon_{1}^{D}-\varepsilon_{2}^{D}\right)\right|^{2}\right)\left|\left(\varepsilon_{2}^{D}+t\left(\varepsilon_{1}^{D}-\varepsilon_{2}^{D}\right)\right) \cdot\left(\varepsilon_{1}-\varepsilon_{2}\right)\right|^{2}\right. \\
& \left.+\psi\left(\left|\varepsilon_{2}^{D}+t\left(\varepsilon_{1}^{D}-\varepsilon_{2}^{D}\right)\right|^{2}\right)\left|\varepsilon_{1}-\varepsilon_{2}\right|^{2}\right] d t \\
\geq & K_{0}\left|\operatorname{tr}\left(\varepsilon_{1}-\varepsilon_{2}\right)\right|^{2} \\
& +\int_{0}^{1}\left[2 \psi^{\prime}\left(\left|\varepsilon_{2}^{D}+t\left(\varepsilon_{1}^{D}-\varepsilon_{2}^{D}\right)\right|^{2}\right)\left|\varepsilon_{2}^{D}+t\left(\varepsilon_{1}^{D}-\varepsilon_{2}^{D}\right)\right|^{2}\right. \\
& \left.+\psi\left(\left|\varepsilon_{2}^{D}+t\left(\varepsilon_{1}^{D}-\varepsilon_{2}^{D}\right)\right|^{2}\right)\right]\left|\varepsilon_{1}-\varepsilon_{2}\right|^{2} d t \\
\geq & K_{0}\left|\operatorname{tr}\left(\varepsilon_{1}-\varepsilon_{2}\right)\right|^{2}+c_{2}\left|\varepsilon_{1}-\varepsilon_{2}\right|^{2} .
\end{aligned}
$$

Hence, the condition (3.13)(b) is satisfied with $m$ depending on $K_{0}$ and $c_{2}$. Conditions (3.13)(c), (d) are obviously valid.

Using the condition (3.13), we see that for all $\boldsymbol{\tau} \in \mathcal{H}$ the function $\boldsymbol{x} \mapsto$ $F(\boldsymbol{x}, \boldsymbol{\tau}(\boldsymbol{x}))$ belongs to $\mathcal{H}$ and hence we may consider $F$ as an operator defined 
on $\mathcal{H}$ with range in $\mathcal{H}$. Moreover, $F: \tau \in \mathcal{H} \mapsto F(\cdot, \tau) \in \mathcal{H}$ is a strongly monotone Lipschitz continuous operator and therefore $F$ is invertible and its inverse $F^{-1}: \mathcal{H} \rightarrow \mathcal{H}$ is also a strongly monotone Lipschitz continuous operator.

We assume the normal compliance functions

$$
p_{r}: \Gamma_{3} \times \mathbb{R} \rightarrow \mathbb{R}_{+} \quad(r=\nu, \tau)
$$

satisfy the following hypothesis for $r=\nu, \tau$.

(3.15) (a) There exists an $L_{r}>0$ such that $\left|p_{r}\left(\boldsymbol{x}, t_{1}\right)-p_{r}\left(\boldsymbol{x}, t_{2}\right)\right| \leq L_{r}\left|t_{1}-t_{2}\right|$ for all $t_{1}, t_{2} \in \mathbb{R}$, a.e. on $\Gamma_{3}$.

(b) The mapping $\boldsymbol{x} \mapsto p_{r}(\boldsymbol{x}, t)$ is Lebesgue measurable on $\Gamma_{3}$ for any $t \in \mathbb{R}$.

(c) $\boldsymbol{x} \mapsto p_{r}(\boldsymbol{x}, t)=0$ for $t \leq 0$.

We observe that the assumptions (3.15) on the functions $p_{\nu}$ and $p_{\tau}$ are fairly general. The most severe restriction comes from the condition (a), which, roughly speaking, requires the functions to grow at most linearly. Certainly the function defined in (3.6) satisfies (3.15)(a), whereas that defined in (3.3) satisfies it if and only if $m_{\nu}=1$. We also observe that if the functions $p_{\nu}$ and $p_{\tau}$ are related by (3.5) and $p_{\nu}$ satisfies (3.15)(a), then so does $p_{\tau}$ with $L_{\tau}=\mu L_{\nu}$. It can be verified that this statement is still valid if the functions $p_{\nu}$ and $p_{\tau}$ are related by (3.7).

The condition $(3.15)(\mathrm{c})$ shows that when there is no penetration (i.e. $\left.u_{\nu} \leq g\right)$ then the tractions vanish $\left(\sigma_{\nu}=0, \boldsymbol{\sigma}_{\tau}=\mathbf{0}\right)$. This condition is satisfied for the function (3.3) if $m_{\nu}>0$. We also observe that if the functions $p_{\nu}$ and $p_{\tau}$ are related by (3.5) or (3.7) and $p_{\nu}$ satisfies (3.15)(c), then so does $p_{\tau}$.

We also suppose that the forces and the tractions have the regularity

$$
\varphi_{1} \in H, \quad \varphi_{2} \in L^{2}\left(\Gamma_{2}\right)^{N}
$$

while the gap function $g$ is such that

$$
g \in L^{\infty}\left(\Gamma_{3}\right), \quad g(\boldsymbol{x}) \geq 0 \quad \text { a.e. on } \Gamma_{3} .
$$

Next, using Riesz's representation theorem, we define $f \in V$ by

$$
(\boldsymbol{f}, \boldsymbol{v})_{V}=\left(\boldsymbol{\varphi}_{1}, \boldsymbol{v}\right)_{H}+\left(\boldsymbol{\varphi}_{2}, \gamma \boldsymbol{v}\right)_{L^{2}\left(\Gamma_{2}\right)^{N}} \quad \forall \boldsymbol{v} \in V
$$

and let $j: V \times V \rightarrow \mathbb{R}$ be the functional

$$
j(\boldsymbol{\eta}, \boldsymbol{v})=\int_{\Gamma_{3}} p_{\nu}\left(\eta_{\nu}-g\right) v_{\nu} d a+\int_{\Gamma_{3}} p_{\tau}\left(\eta_{\nu}-g\right)\left|\boldsymbol{v}_{\tau}\right| d a .
$$

For all $\boldsymbol{\eta} \in V$, set

$$
\Sigma(\boldsymbol{\eta})=\left\{\boldsymbol{\sigma} \in \mathcal{H} \mid(\boldsymbol{\sigma}, \boldsymbol{\varepsilon}(\boldsymbol{v}))_{\mathcal{H}}+j(\boldsymbol{\eta}, \boldsymbol{v}) \geq(\boldsymbol{f}, \boldsymbol{v})_{V} \forall \boldsymbol{v} \in V\right\}
$$

and 


$$
D(T)=\{\boldsymbol{z} \in \mathcal{H} \mid \exists \boldsymbol{v} \in V \text { such that } F(\boldsymbol{\varepsilon}(\boldsymbol{v}))=\boldsymbol{z}\} .
$$

From (3.13)(b) and Korn's inequality (2.5) it follows that the operator $F \circ \varepsilon$ : $V \rightarrow D(T)$ is invertible; we let $T: D(T) \rightarrow V$ denote its inverse. We have

$$
\boldsymbol{v}=T(\boldsymbol{z}) \Leftrightarrow F(\varepsilon(\boldsymbol{v}))=\boldsymbol{z} .
$$

Moreover we obtain the following result.

LEMMA 3.1. If $\{\boldsymbol{u}, \boldsymbol{\sigma}\}$ are sufficiently smooth functions satisfying (3.8)(3.12), then

$$
\begin{aligned}
& \boldsymbol{u} \in V, \\
& (\boldsymbol{\sigma}, \boldsymbol{\varepsilon}(\boldsymbol{v})-\boldsymbol{\varepsilon}(\boldsymbol{u}))_{\mathcal{H}}+j(\boldsymbol{u}, \boldsymbol{v})-j(\boldsymbol{u}, \boldsymbol{u}) \geq(\boldsymbol{f}, \boldsymbol{v}-\boldsymbol{u})_{V} \quad \forall \boldsymbol{v} \in V, \\
& \boldsymbol{\sigma} \in D(T) \cap \Sigma(\boldsymbol{u}), \quad(\boldsymbol{\tau}-\boldsymbol{\sigma}, \boldsymbol{\varepsilon}(\boldsymbol{u}))_{\mathcal{H}} \geq 0 \quad \forall \boldsymbol{\tau} \in \Sigma(\boldsymbol{u}) .
\end{aligned}
$$

Pro of. The regularity $\boldsymbol{u} \in V$ follows from (3.10). Let $\boldsymbol{v} \in V$. Using (2.2), (2.4), (3.9)-(3.11) we have

$$
(\boldsymbol{\sigma}, \boldsymbol{\varepsilon}(\boldsymbol{v}))_{\mathcal{H}}=\left(\boldsymbol{\varphi}_{1}, \boldsymbol{v}\right)_{V}+\left(\boldsymbol{\varphi}_{2}, \gamma \boldsymbol{v}\right)_{L^{2}\left(\Gamma_{2}\right)^{N}}+\int_{\Gamma_{3}} \boldsymbol{\sigma} \boldsymbol{\nu} \cdot \boldsymbol{v} d a,
$$

and using (2.1), (2.3), (3.12) and (3.19) gives

$$
\int_{\Gamma_{3}} \boldsymbol{\sigma \nu} \cdot \boldsymbol{v} d a \geq-j(\boldsymbol{u}, \boldsymbol{v})
$$

Therefore, by (3.25), (3.26) and (3.18) we deduce

$$
(\boldsymbol{\sigma}, \boldsymbol{\varepsilon}(\boldsymbol{v}))_{\mathcal{H}}+j(\boldsymbol{u}, \boldsymbol{v}) \geq(\boldsymbol{f}, \boldsymbol{v})_{V}
$$

The regularity $\boldsymbol{\sigma} \in D(T) \cap \Sigma(\boldsymbol{u})$ now follows from (3.8), (3.21), (3.20) and (3.27).

Moreover, from (3.12) and (3.19) we have

$$
\int_{\Gamma_{3}} \boldsymbol{\sigma} \boldsymbol{\nu} \cdot \boldsymbol{u} d a=-j(\boldsymbol{u}, \boldsymbol{u})
$$

and therefore, taking $\boldsymbol{v}=\boldsymbol{u}$ in (3.25) and using again (3.18), we deduce

$$
(\boldsymbol{\sigma}, \boldsymbol{\varepsilon}(\boldsymbol{u}))_{\mathcal{H}}+j(\boldsymbol{u}, \boldsymbol{u})=(\boldsymbol{f}, \boldsymbol{u})_{V}
$$

The inequalities (3.23) and (3.24) are now a consequence of (3.27), (3.28) and (3.20).

Lemma 3.1, (3.8) and (3.22) lead us to consider the following two variational problems.

Problem $P_{1}$. Find a displacement field $\boldsymbol{u}: \Omega \rightarrow \mathbb{R}^{N}$ such that

$$
\begin{array}{r}
\boldsymbol{u} \in V, \quad(F(\boldsymbol{\varepsilon}(\boldsymbol{u})), \boldsymbol{\varepsilon}(\boldsymbol{v})-\boldsymbol{\varepsilon}(\boldsymbol{u}))_{\mathcal{H}}+j(\boldsymbol{u}, \boldsymbol{v})-j(\boldsymbol{u}, \boldsymbol{u}) \\
\geq(\boldsymbol{f}, \boldsymbol{v}-\boldsymbol{u})_{V} \quad \forall \boldsymbol{v} \in V .
\end{array}
$$


Problem $P_{2}$. Find a stress field $\sigma: \Omega \rightarrow S_{N}$ such that

$$
\boldsymbol{\sigma} \in D(T) \cap \Sigma(T(\boldsymbol{\sigma})), \quad\left(F^{-1}(\boldsymbol{\sigma}), \boldsymbol{\tau}-\boldsymbol{\sigma}\right)_{\mathcal{H}} \geq 0 \quad \forall \boldsymbol{\tau} \in \Sigma(T(\boldsymbol{\sigma})) .
$$

Problems $P_{1}$ and $P_{2}$ are formally equivalent to the mechanical problem $P$. Indeed, if $\boldsymbol{u}$ represents a sufficiently regular solution of $P_{1}$ and $\boldsymbol{\sigma}$ is defined by $\boldsymbol{\sigma}=F(\varepsilon(\boldsymbol{u}))$, then, using the arguments of [6], it follows that $\{\boldsymbol{u}, \boldsymbol{\sigma}\}$ is a solution of problem $P$. Similarly, if $\boldsymbol{\sigma}$ represents a regular solution of $P_{2}$ and $\boldsymbol{u} \in V$ is given by $\boldsymbol{\sigma}=F(\varepsilon(\boldsymbol{u}))$ then, using the same arguments, it follows that $\{\boldsymbol{u}, \boldsymbol{\sigma}\}$ is a solution of $P$. For this reason we may consider problems $P_{1}$ and $P_{2}$ as variational formulations of the mechanical problem $P$.

4. Existence and uniqueness. The main results of this section are on the existence and uniqueness for the two weak formulations $P_{1}$ and $P_{2}$. We have:

TheOREM 4.1. Let the conditions (3.13), (3.15)-(3.17) hold. Then there exists $L_{0}>0$ depending only on $\Omega, \Gamma_{1}$ and $F$ such that if $L_{\nu}+L_{\tau}<L_{0}$ then there exists a unique solution $\boldsymbol{u}$ to problem $P_{1}$.

Theorem 4.2. Let the conditions (3.13), (3.15)-(3.17) hold. Let $L_{0}>0$ be defined as in Theorem 4.1. If $L_{\nu}+L_{\tau}<L_{0}$ then there exists a unique solution $\boldsymbol{\sigma}$ to problem $P_{2}$. Moreover, $\boldsymbol{\sigma} \in \mathcal{H}_{1}$.

The proof of Theorem 4.1 will be carried out in several steps. It is based on fixed point arguments similar to those used in $[14,15]$. We suppose in the sequel that the assumptions of Theorem 4.1 are fulfilled and let $\boldsymbol{\eta} \in V$. We consider the following variational problem.

Problem $P_{1}^{\eta}$. Find $\boldsymbol{u}_{\eta} \in V$ such that

$$
\begin{aligned}
\left(F\left(\boldsymbol{\varepsilon}\left(\boldsymbol{u}_{\eta}\right)\right), \boldsymbol{\varepsilon}(\boldsymbol{v})-\boldsymbol{\varepsilon}\left(\boldsymbol{u}_{\eta}\right)\right)_{\mathcal{H}}+j(\boldsymbol{\eta}, \boldsymbol{v})-j\left(\boldsymbol{\eta}, \boldsymbol{u}_{\eta}\right) & \\
& \geq\left(\boldsymbol{f}, \boldsymbol{v}-\boldsymbol{u}_{\eta}\right)_{V} \quad \forall \boldsymbol{v} \in V .
\end{aligned}
$$

We have the following result.

Lemma 4.3. For any $\boldsymbol{\eta} \in V$, problem $P_{1}^{\eta}$ has a unique solution $\boldsymbol{u}_{\eta} \in V$.

P r o of. Using Riesz's representation theorem we may define an operator $A: V \rightarrow V$ by

$$
(A(\boldsymbol{w}), \boldsymbol{v})_{V}=(F(\varepsilon(\boldsymbol{w})), \boldsymbol{\varepsilon}(\boldsymbol{v}))_{\mathcal{H}} \quad \forall \boldsymbol{w}, \boldsymbol{v} \in V .
$$

Keeping in mind (3.13)(a) we see that $A$ is a Lipschitz continuous operator. Using (3.13)(b) and Korn's inequality (2.5) we conclude that $A$ is a strongly monotone operator. Moreover, by (3.19) it follows that $j(\boldsymbol{\eta}, \cdot)$ is a continuous seminorm on $V$. Lemma 4.3 now results from (4.2) and standard arguments of elliptic variational inequalities. 
Lemma 4.3 allows us to consider an operator $\Lambda: V \rightarrow V$ defined by

$$
\Lambda(\boldsymbol{\eta})=\boldsymbol{u}_{\eta} \quad \forall \boldsymbol{\eta} \in V .
$$

Lemma 4.4. There exists $L_{0}>0$ depending only on $\Omega, \Gamma_{1}$ and $F$ such that if $L_{\nu}+L_{\tau}<L_{0}$ then the operator $\Lambda$ has a unique fixed point $\boldsymbol{\eta}^{*} \in V$.

Proof. We use the Banach fixed point theorem. Let $\boldsymbol{\eta}_{1}, \boldsymbol{\eta}_{2} \in V$ be given and denote the corresponding solutions of the problem (4.1) by $\boldsymbol{u}_{1}$ and $\boldsymbol{u}_{2}$. Then we have $\boldsymbol{u}_{1} \in V, \boldsymbol{u}_{2} \in V$, and

$$
\begin{array}{ll}
\left(F\left(\boldsymbol{\varepsilon}\left(\boldsymbol{u}_{1}\right)\right), \boldsymbol{\varepsilon}(\boldsymbol{v})-\boldsymbol{\varepsilon}\left(\boldsymbol{u}_{1}\right)\right)_{\mathcal{H}}+j\left(\boldsymbol{\eta}_{1}, \boldsymbol{v}\right)-j\left(\boldsymbol{\eta}_{1}, \boldsymbol{u}_{1}\right) \geq\left(\boldsymbol{f}, \boldsymbol{v}-\boldsymbol{u}_{1}\right)_{V} & \forall \boldsymbol{v} \in V, \\
\left(F\left(\boldsymbol{\varepsilon}\left(\boldsymbol{u}_{2}\right)\right), \boldsymbol{\varepsilon}(\boldsymbol{v})-\boldsymbol{\varepsilon}\left(\boldsymbol{u}_{2}\right)\right)_{\mathcal{H}}+j\left(\boldsymbol{\eta}_{2}, \boldsymbol{v}\right)-j\left(\boldsymbol{\eta}_{2}, \boldsymbol{u}_{2}\right) \geq\left(\boldsymbol{f}, \boldsymbol{v}-\boldsymbol{u}_{2}\right)_{V} & \forall \boldsymbol{v} \in V .
\end{array}
$$

We take $\boldsymbol{v}=\boldsymbol{u}_{2}$ in the first inequality, $\boldsymbol{v}=\boldsymbol{u}_{1}$ in the second, and add the two inequalities to obtain

$$
\begin{aligned}
\left(F\left(\varepsilon\left(\boldsymbol{u}_{1}\right)\right)-F(\right. & \left.\left.\varepsilon\left(\boldsymbol{u}_{2}\right)\right), \boldsymbol{\varepsilon}\left(\boldsymbol{u}_{1}\right)-\boldsymbol{\varepsilon}\left(\boldsymbol{u}_{2}\right)\right)_{\mathcal{H}} \\
\leq & j\left(\boldsymbol{\eta}_{1}, \boldsymbol{u}_{2}\right)-j\left(\boldsymbol{\eta}_{1}, \boldsymbol{u}_{1}\right)+j\left(\boldsymbol{\eta}_{2}, \boldsymbol{u}_{1}\right)-j\left(\boldsymbol{\eta}_{2}, \boldsymbol{u}_{2}\right) \\
= & \int_{\Gamma_{3}}\left(p_{\nu}\left(\eta_{1 \nu}-g\right)-p_{\nu}\left(\eta_{2 \nu}-g\right)\right)\left(u_{2 \nu}-u_{1 \nu}\right) d a \\
& \quad+\int_{\Gamma_{3}}\left(p_{\tau}\left(\eta_{1 \nu}-g\right)-p_{\tau}\left(\eta_{2 \nu}-g\right)\right)\left(\left|\boldsymbol{u}_{2 \tau}\right|-\left|\boldsymbol{u}_{1 \tau}\right|\right) d a .
\end{aligned}
$$

Thus, using (3.13) and (3.15) we deduce

$$
\begin{aligned}
m\left\|\varepsilon\left(\boldsymbol{u}_{1}-\boldsymbol{u}_{2}\right)\right\|_{\mathcal{H}}^{2} & \leq \int_{\Gamma_{3}}\left|\eta_{1 \nu}-\eta_{2 \nu}\right|\left(L_{\nu}\left|u_{1 \nu}-u_{2 \nu}\right|+L_{\tau}\left|\boldsymbol{u}_{1 \tau}-\boldsymbol{u}_{2 \tau}\right|\right) d a \\
& \leq\left(L_{\nu}+L_{\tau}\right) \int_{\Gamma_{3}}\left|\eta_{1 \nu}-\eta_{2 \nu}\right|\left|\boldsymbol{u}_{1}-\boldsymbol{u}_{2}\right| d a \\
& \leq\left(L_{\nu}+L_{\tau}\right)\left\|\boldsymbol{\eta}_{1}-\boldsymbol{\eta}_{2}\right\|_{L^{2}\left(\Gamma_{3}\right)^{N}}\left\|\boldsymbol{u}_{1}-\boldsymbol{u}_{2}\right\|_{L^{2}\left(\Gamma_{3}\right)^{N}}
\end{aligned}
$$

By the Sobolev trace theorem and the fact that $\|\varepsilon(\boldsymbol{v})\|_{\mathcal{H}}$ is an equivalent norm on $V$, we have a constant $c_{0}$ depending only on the domain $\Omega$ and $\Gamma_{1}$ such that

$$
\|\boldsymbol{v}\|_{L^{2}\left(\Gamma_{3}\right)^{N}} \leq c_{0}\|\boldsymbol{\varepsilon}(\boldsymbol{v})\|_{\mathcal{H}} \quad \forall \boldsymbol{v} \in V .
$$

Hence,

$$
m\left\|\varepsilon\left(\boldsymbol{u}_{1}-\boldsymbol{u}_{2}\right)\right\|_{\mathcal{H}}^{2} \leq\left(L_{\nu}+L_{\tau}\right) c_{0}^{2}\left\|\varepsilon\left(\boldsymbol{\eta}_{1}-\boldsymbol{\eta}_{2}\right)\right\|_{\mathcal{H}}\left\|\varepsilon\left(\boldsymbol{u}_{1}-\boldsymbol{u}_{2}\right)\right\|_{\mathcal{H}},
$$

i.e.,

Let

$$
\left\|\varepsilon\left(\boldsymbol{u}_{1}-\boldsymbol{u}_{2}\right)\right\|_{\mathcal{H}} \leq \frac{c_{0}^{2}}{m}\left(L_{\nu}+L_{\tau}\right)\left\|\varepsilon\left(\boldsymbol{\eta}_{1}-\boldsymbol{\eta}_{2}\right)\right\|_{\mathcal{H}}
$$

$$
L_{0}=m / c_{0}^{2}
$$


Then if $L_{\nu}+L_{\tau}<L_{0}$, the mapping $\Lambda$ is a contraction of $V$. By the Banach fixed point theorem, the mapping $\Lambda$ has a unique fixed point on $V$.

Proof of Theorem 4.1. Let $L_{\nu}+L_{\tau}<L_{0}$ and let $\boldsymbol{\eta}^{*}$ be the fixed point of the operator $\Lambda$. We denote by $\boldsymbol{u}^{*}$ the solution of the variational problem $P_{1}^{\eta}$ for $\boldsymbol{\eta}=\boldsymbol{\eta}^{*}$. Using (4.1) and (4.3) it is straightforward to see that $\boldsymbol{u}^{*}$ is a solution of (3.29). This proves the existence part of Theorem 4.1. The uniqueness part follows from the uniqueness of the fixed point of the operator $\Lambda$ given by (4.3).

Next, we present the proof of Theorem 4.2. For this we suppose in the sequel that conditions (3.13), (3.15)-(3.17) hold and let again $\boldsymbol{\eta} \in V$. We consider the following variational problem.

Problem $P_{2}^{\eta}$. Find $\sigma_{\eta}: \Omega \rightarrow S_{N}$ such that

$$
\boldsymbol{\sigma}_{\eta} \in D(T) \cap \Sigma(\boldsymbol{\eta}), \quad\left(F^{-1}\left(\boldsymbol{\sigma}_{\eta}\right), \boldsymbol{\tau}-\boldsymbol{\sigma}_{\eta}\right)_{\mathcal{H}} \geq 0 \quad \forall \boldsymbol{\tau} \in \Sigma(\boldsymbol{\eta}) .
$$

We have the following result.

Lemma 4.5. For any $\boldsymbol{\eta} \in V$, problem $P_{2}^{\eta}$ has a unique solution $\boldsymbol{\sigma}_{\eta}$. Moreover, $\sigma_{\eta} \in \mathcal{H}_{1}$.

Proof. Let $\boldsymbol{\sigma}_{\eta} \in D(T)$ be given by

$$
\boldsymbol{\sigma}_{\eta}=F\left(\varepsilon\left(\boldsymbol{u}_{\eta}\right)\right)
$$

where $\boldsymbol{u}_{\eta}$ is the solution of problem $P_{1}^{\eta}$. Taking $\boldsymbol{v}=2 \boldsymbol{u}_{\eta}$ and $\boldsymbol{v}=\mathbf{0}$ in (4.1) we get

$$
\left(\boldsymbol{\sigma}_{\eta}, \boldsymbol{\varepsilon}\left(\boldsymbol{u}_{\eta}\right)\right)_{\mathcal{H}}+j\left(\boldsymbol{\eta}, \boldsymbol{u}_{\eta}\right)=\left(\boldsymbol{f}, \boldsymbol{u}_{\eta}\right)_{V} .
$$

Moreover, from (4.1), (4.7) and (4.8) we deduce that

$$
\left(\boldsymbol{\sigma}_{\eta}, \boldsymbol{\varepsilon}(\boldsymbol{v})\right)_{\mathcal{H}}+j(\boldsymbol{\eta}, \boldsymbol{v}) \geq(\boldsymbol{f}, \boldsymbol{v})_{V} \quad \forall \boldsymbol{v} \in V,
$$

which implies $\boldsymbol{\sigma}_{\eta} \in \Sigma(\boldsymbol{\eta})$. Using again (4.7), (3.20) and (4.8) we see that

$$
\left(F^{-1}\left(\boldsymbol{\sigma}_{\eta}\right), \boldsymbol{\tau}-\boldsymbol{\sigma}_{\eta}\right)_{\mathcal{H}}=\left(\boldsymbol{\tau}-\boldsymbol{\sigma}_{\eta}, \boldsymbol{\varepsilon}\left(\boldsymbol{u}_{\eta}\right)\right)_{\mathcal{H}} \geq 0 \quad \forall \boldsymbol{\tau} \in \Sigma(\boldsymbol{\eta}),
$$

which proves the existence part in Lemma 4.5. The uniqueness part follows from standard arguments since $F^{-1}: \mathcal{H} \rightarrow \mathcal{H}$ is a strongly monotone operator.

The regularity $\boldsymbol{\sigma}_{\eta} \in \mathcal{H}_{1}$ follows from $\boldsymbol{\sigma}_{\eta} \in \Sigma(\boldsymbol{\eta})$. Indeed, taking $\boldsymbol{v}= \pm \boldsymbol{\varphi}$ with $\varphi \in \mathcal{D}(\Omega)^{N}$ in the constraint inequality defining the set (3.20) and using (3.18), (3.19) we deduce

$$
\operatorname{Div} \boldsymbol{\sigma}_{\eta}+\boldsymbol{\varphi}_{1}=\mathbf{0} \quad \text { a.e. in } \Omega \text {. }
$$

Then with the condition (3.16), it follows that $\boldsymbol{\sigma}_{\eta} \in \mathcal{H}_{1}$.

Proof of Theorem 4.2. Let $L_{\nu}+L_{\tau}<L_{0}$ and let $\boldsymbol{\eta}^{*}$ be the fixed point of the operator $\Lambda$ defined by (4.3). We denote by $\boldsymbol{\sigma}^{*}$ the solution of the variational problem $P_{2}^{\eta}$ for $\boldsymbol{\eta}=\boldsymbol{\eta}^{*}$. From (4.7) and (3.22) it follows that 
$\boldsymbol{u}^{*}=T\left(\boldsymbol{\sigma}^{*}\right)$ where $\boldsymbol{u}^{*}$ is the solution of $P_{1}^{\eta}$ for $\boldsymbol{\eta}=\boldsymbol{\eta}^{*}$. Moreover, by (4.3) we have

$$
\boldsymbol{\eta}^{*}=T\left(\boldsymbol{\sigma}^{*}\right) \text {. }
$$

From (4.6) and (4.10) it now follows that $\boldsymbol{\sigma}^{*}$ satisfies (3.30), which proves the existence part of Theorem 4.2. The regularity $\sigma^{*} \in \mathcal{H}_{1}$ follows from Lemma 4.5.

In order to prove the uniqueness part let $\sigma^{*}$ be the solution of problem $P_{2}$ obtained above and let $\boldsymbol{\sigma}$ be another solution of $P_{2}$. We denote by $\boldsymbol{\eta}$ the element of $V$ given by

$$
\boldsymbol{\eta}=T(\boldsymbol{\sigma}) .
$$

From (3.30) and (4.11) it follows that $\boldsymbol{\sigma}$ is a solution of problem $P_{2}^{\eta}$ and, since by Lemma 4.5 this problem has the unique solution $\boldsymbol{\sigma}_{\eta}$ given by (4.7), we have

$$
\sigma=\sigma_{\eta} .
$$

Using again (4.7) and (3.22) we get

$$
\boldsymbol{u}_{\eta}=T\left(\boldsymbol{\sigma}_{\eta}\right)
$$

and therefore, by (4.11)-(4.13), it follows that $\boldsymbol{u}_{\eta}=\boldsymbol{\eta}$. We conclude that $\boldsymbol{\eta}$ is the fixed point of the operator $\Lambda$ given by (4.3), and by Lemma 4.4 it follows that

$$
\boldsymbol{\eta}=\boldsymbol{\eta}^{*} \text {. }
$$

The uniqueness part in Theorem 4.1 is now a consequence of (4.12) and (4.14).

As was pointed out in Section 3 , problems $P_{1}$ and $P_{2}$ represent two variational formulations of the mechanical problem $P$. Problem $P_{1}$ is formulated in terms of displacements while problem $P_{2}$ is formulated in terms of stresses. We are interested in the relation between the solutions $\boldsymbol{u}$ and $\boldsymbol{\sigma}$ obtained in Theorems 4.1 and 4.2, which is stated in the next result.

TheOrem 4.6. Let the conditions (3.13), (3.15)-(3.17) hold, and assume $L_{\nu}+L_{\tau}<L_{0}$.

1) Let $\boldsymbol{u}$ be the solution of problem $P_{1}$ and $\boldsymbol{\sigma}$ be given by

$$
\boldsymbol{\sigma}=F(\varepsilon(\boldsymbol{u})) .
$$

Then $\boldsymbol{\sigma}$ belongs to $\mathcal{H}_{1}$ and $\boldsymbol{\sigma}$ is the solution of problem $P_{2}$.

2) Conversely, let $\boldsymbol{\sigma}$ be the solution of problem $P_{2}$. Then there exists a unique $\boldsymbol{u} \in V$ such that (4.15) holds and $\boldsymbol{u}$ is the solution of problem $P_{1}$.

Proof. 1) Let $\boldsymbol{u}$ be the solution of problem $P_{1}$. It follows from the proof of Theorem 4.1 that $\boldsymbol{u}$ is the solution of problem $P_{1}^{\eta^{*}}$ where $\eta^{*}$ is the 
fixed point of the operator $\Lambda$ given by (4.3). Therefore, from the proof of Lemma 4.5 we deduce that $\boldsymbol{\sigma}$ given by $(4.15)$ is a solution of $P_{2}^{\eta^{*}}$, which concludes the proof.

2) Conversely, let $\boldsymbol{\sigma}$ be the solution of $P_{2}$ and let $\boldsymbol{u}=T(\boldsymbol{\sigma}) \in V$. Using (3.22) we deduce that (4.15) holds and moreover

$$
(\boldsymbol{\tau}-\boldsymbol{\sigma}, \boldsymbol{\varepsilon}(\boldsymbol{u}))_{\mathcal{H}} \geq \mathbf{0} \quad \forall \boldsymbol{\tau} \in \Sigma(\boldsymbol{u}) .
$$

Using now the subdifferentiability of the seminorm $j(\boldsymbol{u}, \cdot)$ on $V$ and (2.6) we deduce that there exists $\widetilde{\tau} \in \mathcal{H}$ such that

$$
(\widetilde{\boldsymbol{\tau}}, \boldsymbol{\varepsilon}(\boldsymbol{v})-\varepsilon(\boldsymbol{u}))_{\mathcal{H}}+j(\boldsymbol{u}, \boldsymbol{v})-j(\boldsymbol{u}, \boldsymbol{u}) \geq(\boldsymbol{f}, \boldsymbol{v}-\boldsymbol{u})_{V} \quad \forall \boldsymbol{v} \in V .
$$

Taking $\boldsymbol{v}=2 \boldsymbol{u}$ and $\boldsymbol{v}=\mathbf{0}$ in (4.17) we obtain

$$
(\widetilde{\boldsymbol{\tau}}, \boldsymbol{\varepsilon}(\boldsymbol{u}))_{\mathcal{H}}+j(\boldsymbol{u}, \boldsymbol{u})=(\boldsymbol{f}, \boldsymbol{u})_{V}
$$

From (4.17), (4.18) and (3.20), it now follows that $\tilde{\boldsymbol{\tau}} \in \Sigma(\boldsymbol{u})$. Therefore, taking $\boldsymbol{\tau}=\widetilde{\boldsymbol{\tau}}$ in (4.16) and using again (4.18) we deduce

$$
(\boldsymbol{f}, \boldsymbol{u})_{V} \geq(\boldsymbol{\sigma}, \boldsymbol{\varepsilon}(\boldsymbol{u}))_{\mathcal{H}}+j(\boldsymbol{u}, \boldsymbol{u}) .
$$

The converse inequality follows from (3.20) since $\boldsymbol{\sigma} \in \Sigma(T(\boldsymbol{\sigma}))$ and $T(\boldsymbol{\sigma})=$ $\boldsymbol{u}$. Therefore, we conclude that

$$
(\boldsymbol{\sigma}, \boldsymbol{\varepsilon}(\boldsymbol{u}))_{\mathcal{H}}+j(\boldsymbol{u}, \boldsymbol{u})=(\boldsymbol{f}, \boldsymbol{u})_{V} .
$$

Using again (3.20) we have

$$
(\boldsymbol{\sigma}, \boldsymbol{\varepsilon}(\boldsymbol{v}))_{\mathcal{H}}+j(\boldsymbol{u}, \boldsymbol{v}) \geq(\boldsymbol{f}, \boldsymbol{v})_{V}
$$

and from (4.20), (4.19) and (4.15) it results that $\boldsymbol{u}$ is a solution of $P_{1}$.

The mechanical interpretation of the results in Theorem 4.6 is the following.

1) If the displacement field $\boldsymbol{u}$ is the solution of the variational problem $P_{1}$, then the associated stress field $\boldsymbol{\sigma}=F(\boldsymbol{\varepsilon}(\boldsymbol{u}))$ is the solution of the variational problem $P_{2}$.

2) If the stress field $\sigma$ is the solution of $P_{2}$ then there exists a displacement field $\boldsymbol{u} \in V$ associated with $\boldsymbol{\sigma}$ by the elastic constitutive law $\boldsymbol{\sigma}=F(\varepsilon(\boldsymbol{u}))$ and $\boldsymbol{u}$ is the solution of $P_{1}$.

Under the assumptions of Theorems 4.1 and 4.2, we also see that if the displacement field $\boldsymbol{u}$ is the solution of $P_{1}$ and the stress field $\boldsymbol{\sigma}$ is the solution of $P_{2}$, then $\boldsymbol{u}$ and $\boldsymbol{\sigma}$ are connected by the elastic constitutive law $\boldsymbol{\sigma}=F(\varepsilon(\boldsymbol{u}))$. For this reason we shall consider in the sequel the couple $\{\boldsymbol{u}, \boldsymbol{\sigma}\}$ given by Theorems 4.1 and 4.2 as a weak solution for the problem (3.8)-(3.12) and we conclude that this mechanical problem has a unique weak solution provided $L_{\nu}+L_{\tau}<L_{0}$. 
5. Continuous dependence on contact conditions. Next, we investigate the behavior of the weak solution to the problem (3.8)-(3.12) with respect to perturbations of the normal compliance functions $p_{\nu}$ and $p_{\tau}$. To this end, suppose that the conditions (3.13), (3.15)-(3.17) hold. For every $\alpha \geq 0$, let $p_{r}^{\alpha}$ be a perturbation of $p_{r}$ which satisfies (3.15) with Lipschitz constant $L_{r}^{\alpha}(r=\nu, \tau)$. Let us also introduce the functionals $j^{\alpha}$ which are obtained by replacing $p_{\nu}$ and $p_{\tau}$ by $p_{\nu}^{\alpha}$ and $p_{\tau}^{\alpha}$ in $j$, and let $\Sigma^{\alpha}(\boldsymbol{\eta})$ be given by (3.20) with $j$ replaced by $j^{\alpha}$. We now consider the following problems.

Problem $P_{1}^{\alpha}$. For $\alpha \geq 0$, find a displacement field $\boldsymbol{u}^{\alpha} \in V$ such that

$$
\begin{aligned}
&\left(F\left(\varepsilon\left(\boldsymbol{u}^{\alpha}\right)\right), \boldsymbol{\varepsilon}(\boldsymbol{v})-\boldsymbol{\varepsilon}\left(\boldsymbol{u}^{\alpha}\right)\right)_{\mathcal{H}}+j^{\alpha}\left(\boldsymbol{u}^{\alpha}, \boldsymbol{v}\right)-j^{\alpha}\left(\boldsymbol{u}^{\alpha}, \boldsymbol{u}^{\alpha}\right) \\
& \geq\left(\boldsymbol{f}, \boldsymbol{v}-\boldsymbol{u}^{\alpha}\right)_{V} \quad \forall \boldsymbol{v} \in V .
\end{aligned}
$$

Problem $P_{2}^{\alpha}$. For $\alpha \geq 0$, find a stress field $\boldsymbol{\sigma}^{\alpha} \in D(T) \cap \Sigma^{\alpha}\left(T\left(\boldsymbol{\sigma}^{\alpha}\right)\right)$ such that

$$
\left(F^{-1}\left(\boldsymbol{\sigma}^{\alpha}\right), \boldsymbol{\tau}-\boldsymbol{\sigma}^{\alpha}\right)_{\mathcal{H}} \geq 0 \quad \forall \boldsymbol{\tau} \in \Sigma^{\alpha}\left(T\left(\boldsymbol{\sigma}^{\alpha}\right)\right) .
$$

We suppose that $L_{\nu}^{\alpha}+L_{\tau}^{\alpha}<L_{0}$. Using Theorems 4.1 and 4.2 , we deduce that for each $\alpha \geq 0$, problem $P_{1}^{\alpha}$ has a unique solution $\boldsymbol{u}^{\alpha} \in V$ and problem $P_{2}^{\alpha}$ has a unique solution $\boldsymbol{\sigma}^{\alpha} \in \mathcal{H}_{1}$. Moreover, by Theorem 4.6 it follows that $\boldsymbol{\sigma}^{\alpha}$ and $\boldsymbol{u}^{\alpha}$ are connected by the elastic constitutive law, i.e.,

$$
\boldsymbol{\sigma}^{\alpha}=F\left(\varepsilon\left(\boldsymbol{u}^{\alpha}\right)\right)
$$

Suppose now that the normal compliance functions satisfy the following assumption: there exist $\varphi_{r}: \mathbb{R}_{+} \rightarrow \mathbb{R}_{+}(r=\nu, \tau)$ and $\beta>0$ such that

(5.4) (a) $\left|p_{r}^{\alpha}(x, t)-p_{r}(x, t)\right| \leq \varphi_{r}(\alpha)|t|$ for all $t \in \mathbb{R}$, a.e. on $\Gamma_{3}$.

(b) $\lim _{\alpha \rightarrow 0} \varphi_{r}(\alpha)=0$.

(c) $L_{\nu}^{\alpha}+L_{\tau}^{\alpha}+\beta \leq L_{0}$ for all $\alpha \geq 0$.

Under these assumptions we have the following result.

Theorem 5.1. Let (5.4) hold. Then

$$
\boldsymbol{u}^{\alpha} \rightarrow \boldsymbol{u} \text { in } V, \quad \boldsymbol{\sigma}^{\alpha} \rightarrow \boldsymbol{\sigma} \text { in } \mathcal{H}_{1}, \quad \text { as } \alpha \rightarrow 0 .
$$

Proof. Let $\alpha \geq 0$. Using (3.29) and (5.1) we obtain

$$
\begin{aligned}
\left(F\left(\varepsilon\left(\boldsymbol{u}^{\alpha}\right)\right)-F(\varepsilon(\boldsymbol{u})), \boldsymbol{\varepsilon}\left(\boldsymbol{u}^{\alpha}\right)-\boldsymbol{\varepsilon}(\boldsymbol{u})\right)_{\mathcal{H}} \\
\leq j\left(\boldsymbol{u}, \boldsymbol{u}^{\alpha}\right)-j(\boldsymbol{u}, \boldsymbol{u})+j^{\alpha}\left(\boldsymbol{u}^{\alpha}, \boldsymbol{u}\right)-j^{\alpha}\left(\boldsymbol{u}^{\alpha}, \boldsymbol{u}^{\alpha}\right) \\
=\int_{\Gamma_{3}}\left(p_{\nu}\left(u_{\nu}-g\right)-p_{\nu}^{\alpha}\left(u_{\nu}^{\alpha}-g\right)\right)\left(u_{\nu}^{\alpha}-u_{\nu}\right) d a \\
\quad+\int_{\Gamma_{3}}\left(p_{\tau}\left(u_{\nu}-g\right)-p_{\tau}^{\alpha}\left(u_{\nu}^{\alpha}-g\right)\right)\left(\left|\boldsymbol{u}_{\tau}^{\alpha}\right|-\left|\boldsymbol{u}_{\tau}\right|\right) d a .
\end{aligned}
$$


Thus, using (3.13), we deduce

$$
\begin{aligned}
& \quad m\left\|\boldsymbol{\varepsilon}\left(\boldsymbol{u}^{\alpha}-\boldsymbol{u}\right)\right\|_{\mathcal{H}}^{2} \\
& \leq \int_{\Gamma_{3}}\left\{\left|p_{\nu}\left(u_{\nu}-g\right)-p_{\nu}^{\alpha}\left(u_{\nu}^{\alpha}-g\right)\right|+\left|p_{\tau}\left(u_{\nu}-g\right)-p_{\tau}^{\alpha}\left(u_{\nu}^{\alpha}-g\right)\right|\right\}\left|\boldsymbol{u}^{\alpha}-\boldsymbol{u}\right| d a .
\end{aligned}
$$

Let now $r=\nu$ or $\tau$. Then

$\left|p_{r}\left(u_{\nu}-g\right)-p_{r}^{\alpha}\left(u_{\nu}^{\alpha}-g\right)\right| \leq\left|p_{r}\left(u_{\nu}-g\right)-p_{r}^{\alpha}\left(u_{\nu}-g\right)\right|+\left|p_{r}^{\alpha}\left(u_{\nu}-g\right)-p_{r}^{\alpha}\left(u_{\nu}^{\alpha}-g\right)\right|$

a.e. on $\Gamma_{3}$. Taking into account (5.4)(a) and (3.15), we get

$$
\left|p_{r}\left(u_{\nu}-g\right)-p_{r}^{\alpha}\left(u_{\nu}^{\alpha}-g\right)\right| \leq \varphi_{r}(\alpha)|\boldsymbol{u}|+L_{r}^{\alpha}\left|\boldsymbol{u}^{\alpha}-\boldsymbol{u}\right|
$$

a.e. on $\Gamma_{3}$. Combining now (5.5) and (5.6) we deduce

$$
\begin{aligned}
m\left\|\boldsymbol{\varepsilon}\left(\boldsymbol{u}^{\alpha}-\boldsymbol{u}\right)\right\|_{\mathcal{H}}^{2} & \\
\leq & \int_{\Gamma_{3}}\left[\left(\varphi_{\nu}(\alpha)+\varphi_{\tau}(\alpha)\right)|\boldsymbol{u}|\left|\boldsymbol{u}^{\alpha}-\boldsymbol{u}\right|+\left(L_{\nu}^{\alpha}+L_{\tau}^{\alpha}\right)\left|\boldsymbol{u}^{\alpha}-\boldsymbol{u}\right|^{2}\right] d a \\
\leq & \left(\varphi_{\nu}(\alpha)+\varphi_{\tau}(\alpha)\right)\|\boldsymbol{u}\|_{L^{2}\left(\Gamma_{3}\right)^{N}}\left\|\boldsymbol{u}^{\alpha}-\boldsymbol{u}\right\|_{L^{2}\left(\Gamma_{3}\right)^{N}} \\
& \quad+\left(L_{\nu}^{\alpha}+L_{\tau}^{\alpha}\right)\left\|\boldsymbol{u}^{\alpha}-\boldsymbol{u}\right\|_{L^{2}\left(\Gamma_{3}\right)^{N}}^{2}
\end{aligned}
$$

and by (4.4) it follows that

$$
\begin{aligned}
& m\left\|\varepsilon\left(\boldsymbol{u}^{\alpha}-\boldsymbol{u}\right)\right\|_{\mathcal{H}}^{2} \\
& \quad \leq c_{0}^{2}\left(\varphi_{\nu}(\alpha)+\varphi_{\tau}(\alpha)\right)\|\boldsymbol{\varepsilon}(\boldsymbol{u})\|_{\mathcal{H}}\left\|\varepsilon\left(\boldsymbol{u}^{\alpha}-\boldsymbol{u}\right)\right\|_{\mathcal{H}}+c_{0}^{2}\left(L_{\nu}^{\alpha}+L_{\tau}^{\alpha}\right)\left\|\varepsilon\left(\boldsymbol{u}^{\alpha}-\boldsymbol{u}\right)\right\|_{\mathcal{H}}^{2} .
\end{aligned}
$$

Using now (4.5) and (5.4)(c) in the previous inequality, we deduce

$$
\beta\left\|\varepsilon\left(\boldsymbol{u}^{\alpha}-\boldsymbol{u}\right)\right\|_{\mathcal{H}} \leq\left(\varphi_{\nu}(\alpha)+\varphi_{\tau}(\alpha)\right)\|\varepsilon(\boldsymbol{u})\|_{\mathcal{H}} .
$$

Moreover, from (4.15), (5.3) and (3.13) it follows that

$$
\left\|\boldsymbol{\sigma}^{\alpha}-\boldsymbol{\sigma}\right\|_{\mathcal{H}} \leq M\left\|\varepsilon\left(\boldsymbol{u}^{\alpha}-\boldsymbol{u}\right)\right\|_{\mathcal{H}}
$$

and since Div $\boldsymbol{\sigma}^{\alpha}=\operatorname{Div} \boldsymbol{\sigma}=-\boldsymbol{\varphi}_{1}$ (see (4.9)) we obtain

$$
\left\|\boldsymbol{\sigma}^{\alpha}-\boldsymbol{\sigma}\right\|_{\mathcal{H}_{1}}=\left\|\boldsymbol{\sigma}^{\alpha}-\boldsymbol{\sigma}\right\|_{\mathcal{H}} \leq M\left\|\varepsilon\left(\boldsymbol{u}^{\alpha}-\boldsymbol{u}\right)\right\|_{\mathcal{H}} .
$$

The convergence result (5.5) is now a consequence of (5.7), (5.8) and $(5.4)(\mathrm{b})$.

In addition to the mathematical interest in the result of Theorem 5.1, it is of importance in applications, as it indicates that a small perturbation in the contact condition leads to a small change in the solution.

6. Finite element approximation. In this section we study the finite element approximation of the variational problem $P_{1}$. Everywhere in the sequel $c$ will denote a strictly positive generic constant which may depend on $\Omega, \Gamma_{1}$ and $F$ and whose value may vary from place to place. Let $V_{h} \subset V$ 
be a finite element subspace. Then the finite element solution of problem $P_{1}$ is $\boldsymbol{u}_{h} \in V_{h}$ which satisfies

$$
\begin{aligned}
\left(F\left(\boldsymbol{\varepsilon}\left(\boldsymbol{u}_{h}\right)\right), \boldsymbol{\varepsilon}\left(\boldsymbol{v}_{h}\right)-\boldsymbol{\varepsilon}\left(\boldsymbol{u}_{h}\right)\right)_{\mathcal{H}}+j\left(\boldsymbol{u}_{h}, \boldsymbol{v}_{h}\right)-j\left(\boldsymbol{u}_{h}, \boldsymbol{u}_{h}\right) & \\
& \geq\left(\boldsymbol{f}, \boldsymbol{v}_{h}-\boldsymbol{u}_{h}\right)_{V} \quad \forall \boldsymbol{v}_{h} \in V_{h} .
\end{aligned}
$$

Under the assumptions of Theorem 4.1 with the same value of $L_{0}$, the discrete system (6.1) has a unique solution $\boldsymbol{u}_{h} \in V_{h}$. Here we focus on error analysis of the numerical solution. We first derive a Céa's type inequality.

TheOREM 6.1. Under the assumptions of Theorem 4.1 with the same value of $L_{0}$, for some constant $c>0$ we have

$$
\begin{aligned}
& \quad\left\|\boldsymbol{u}-\boldsymbol{u}_{h}\right\|_{V} \\
& \leq c \inf _{\boldsymbol{v}_{h} \in V_{h}}\left\{\left\|\boldsymbol{u}-\boldsymbol{v}_{h}\right\|_{V}+\left\|\boldsymbol{u}-\boldsymbol{v}_{h}\right\|_{L^{2}\left(\Gamma_{3}\right)^{N}}+\|F(\varepsilon(\boldsymbol{u}))\|_{\mathcal{H}}^{1 / 2}\left\|\boldsymbol{u}-\boldsymbol{v}_{h}\right\|_{V}^{1 / 2}\right. \\
& \left.\quad+\left(\left\|p_{\nu}\left(u_{\nu}-g\right)\right\|_{L^{2}\left(\Gamma_{3}\right)}+\left\|p_{\tau}\left(u_{\nu}-g\right)\right\|_{L^{2}\left(\Gamma_{3}\right)}\right)^{1 / 2}\left\|\boldsymbol{u}-\boldsymbol{v}_{h}\right\|_{L^{2}\left(\Gamma_{3}\right)^{N}}^{1 / 2}\right\} .
\end{aligned}
$$

Proof. First we have

$$
\begin{aligned}
& m\left\|\boldsymbol{\varepsilon}\left(\boldsymbol{u}-\boldsymbol{u}_{h}\right)\right\|_{\mathcal{H}}^{2} \\
& \leq\left(F(\varepsilon(\boldsymbol{u}))-F\left(\varepsilon\left(\boldsymbol{u}_{h}\right)\right), \boldsymbol{\varepsilon}\left(\boldsymbol{u}-\boldsymbol{u}_{h}\right)\right)_{\mathcal{H}} \\
&=\left(F(\boldsymbol{\varepsilon}(\boldsymbol{u}))-F\left(\varepsilon\left(\boldsymbol{u}_{h}\right)\right), \boldsymbol{\varepsilon}\left(\boldsymbol{u}-\boldsymbol{v}_{h}\right)\right)_{\mathcal{H}}+\left(F(\varepsilon(\boldsymbol{u})), \boldsymbol{\varepsilon}\left(\boldsymbol{v}_{h}-\boldsymbol{u}\right)\right)_{\mathcal{H}} \\
& \quad+\left(F(\varepsilon(\boldsymbol{u})), \boldsymbol{\varepsilon}\left(\boldsymbol{u}-\boldsymbol{u}_{h}\right)\right)_{\mathcal{H}}-\left(F\left(\varepsilon\left(\boldsymbol{u}_{h}\right)\right), \boldsymbol{\varepsilon}\left(\boldsymbol{v}_{h}-\boldsymbol{u}_{h}\right)\right)_{\mathcal{H}} .
\end{aligned}
$$

We then use (3.29) with $\boldsymbol{v}=\boldsymbol{u}_{h}$ and (6.1) to get

$$
\begin{aligned}
& m\left\|\boldsymbol{\varepsilon}\left(\boldsymbol{u}-\boldsymbol{u}_{h}\right)\right\|_{\mathcal{H}}^{2} \\
& \leq\left(F(\boldsymbol{\varepsilon}(\boldsymbol{u}))-F\left(\boldsymbol{\varepsilon}\left(\boldsymbol{u}_{h}\right)\right), \boldsymbol{\varepsilon}\left(\boldsymbol{u}-\boldsymbol{v}_{h}\right)\right)_{\mathcal{H}}+\left(F(\varepsilon(\boldsymbol{u})), \boldsymbol{\varepsilon}\left(\boldsymbol{v}_{h}-\boldsymbol{u}\right)_{\mathcal{H}}\right. \\
& \quad+j\left(\boldsymbol{u}, \boldsymbol{u}_{h}\right)-j(\boldsymbol{u}, \boldsymbol{u})+j\left(\boldsymbol{u}_{h}, \boldsymbol{v}_{h}\right)-j\left(\boldsymbol{u}_{h}, \boldsymbol{u}_{h}\right)-\left(\boldsymbol{f}, \boldsymbol{v}_{h}-\boldsymbol{u}\right)_{V},
\end{aligned}
$$

i.e.,

$$
m\left\|\varepsilon\left(\boldsymbol{u}-\boldsymbol{u}_{h}\right)\right\|_{\mathcal{H}}^{2} \leq R_{1}+R_{2}+R_{3}+R_{4},
$$

where

$$
\begin{aligned}
& R_{1}=\left(F(\varepsilon(\boldsymbol{u}))-F\left(\varepsilon\left(\boldsymbol{u}_{h}\right)\right), \boldsymbol{\varepsilon}\left(\boldsymbol{u}-\boldsymbol{v}_{h}\right)\right)_{\mathcal{H}}, \\
& R_{2}=\left(F(\varepsilon(\boldsymbol{u})), \boldsymbol{\varepsilon}\left(\boldsymbol{v}_{h}-\boldsymbol{u}\right)\right)_{\mathcal{H}}+j\left(\boldsymbol{u}, \boldsymbol{v}_{h}\right)-j(\boldsymbol{u}, \boldsymbol{u})-\left(\boldsymbol{f}, \boldsymbol{v}_{h}-\boldsymbol{u}\right)_{V}, \\
& R_{3}=j\left(\boldsymbol{u}, \boldsymbol{u}_{h}\right)-j\left(\boldsymbol{u}_{h}, \boldsymbol{u}_{h}\right)+j\left(\boldsymbol{u}_{h}, \boldsymbol{u}\right)-j(\boldsymbol{u}, \boldsymbol{u}), \\
& R_{4}=j\left(\boldsymbol{u}_{h}, \boldsymbol{v}_{h}\right)-j\left(\boldsymbol{u}, \boldsymbol{v}_{h}\right)+j(\boldsymbol{u}, \boldsymbol{u})-j\left(\boldsymbol{u}_{h}, \boldsymbol{u}\right) .
\end{aligned}
$$

Let us estimate each of the four terms. For the first term, we have

$$
\left|R_{1}\right| \leq M\left\|\varepsilon\left(\boldsymbol{u}-\boldsymbol{u}_{h}\right)\right\|_{\mathcal{H}}\left\|\varepsilon\left(\boldsymbol{u}-\boldsymbol{v}_{h}\right)\right\|_{\mathcal{H}} .
$$


The second term $R_{2}$ can be viewed as a residual, and by a straightforward estimation, we have

$$
\begin{aligned}
\left|R_{2}\right| \leq & \|F(\varepsilon(\boldsymbol{u}))\|_{\mathcal{H}}\left\|\varepsilon\left(\boldsymbol{u}-\boldsymbol{v}_{h}\right)\right\|_{\mathcal{H}} \\
& +\left(\left\|p_{\nu}\left(u_{\nu}-g\right)\right\|_{L^{2}\left(\Gamma_{3}\right)}+\left\|p_{\tau}\left(u_{\nu}-g\right)\right\|_{L^{2}\left(\Gamma_{3}\right)}\right)\left\|\boldsymbol{u}-\boldsymbol{v}_{h}\right\|_{L^{2}\left(\Gamma_{3}\right)^{N}}
\end{aligned}
$$

Since

we have

$$
\begin{aligned}
R_{3}= & \int_{\Gamma_{3}}\left[\left(p_{\nu}\left(u_{\nu}-g\right)-p_{\nu}\left(u_{h \nu}-g\right)\right)\left(u_{h \nu}-u_{\nu}\right)\right. \\
& \left.+\left(p_{\tau}\left(u_{\nu}-g\right)-p_{\tau}\left(u_{h \nu}-g\right)\right)\left(\left|\boldsymbol{u}_{h \tau}\right|-\left|\boldsymbol{u}_{\tau}\right|\right)\right] d a,
\end{aligned}
$$

$$
\left|R_{3}\right| \leq \int_{\Gamma_{3}}\left[L_{\nu}\left|u_{\nu}-u_{h \nu}\right|^{2}+L_{\tau}\left|u_{\nu}-u_{h \nu}\right|\left|\boldsymbol{u}_{\tau}-\boldsymbol{u}_{h \tau}\right|\right] d a
$$

and thus

$$
\left|R_{3}\right| \leq c\left(L_{\nu}+L_{\tau}\right)\left\|\varepsilon\left(\boldsymbol{u}-\boldsymbol{u}_{h}\right)\right\|_{\mathcal{H}}^{2} .
$$

Similarly,

$$
\begin{aligned}
R_{4}= & \int_{\Gamma_{3}}\left[\left(p_{\nu}\left(u_{h \nu}-g\right)-p_{\nu}\left(u_{\nu}-g\right)\right)\left(v_{h \nu}-u_{\nu}\right)\right. \\
& \left.+\left(p_{\tau}\left(u_{h \nu}-g\right)-p_{\tau}\left(u_{\nu}-g\right)\right)\left(\left|\boldsymbol{v}_{h \tau}\right|-\left|\boldsymbol{u}_{\tau}\right|\right)\right] d a
\end{aligned}
$$

and then

$$
\begin{aligned}
\left|R_{4}\right| & \leq \int_{\Gamma_{3}}\left[L_{\nu}\left|u_{\nu}-u_{h \nu}\right|\left|u_{\nu}-v_{h \nu}\right|+L_{\tau}\left|u_{\nu}-u_{h \nu}\right|\left|\boldsymbol{u}_{\tau}-\boldsymbol{v}_{h \tau}\right|\right] d a \\
& \leq c\left\|\boldsymbol{u}-\boldsymbol{u}_{h}\right\|_{L^{2}\left(\Gamma_{3}\right)^{N}}\left\|\boldsymbol{u}-\boldsymbol{v}_{h}\right\|_{L^{2}\left(\Gamma_{3}\right)^{N}} \\
& \leq c\left\|\varepsilon\left(\boldsymbol{u}-\boldsymbol{u}_{h}\right)\right\|_{\mathcal{H}}\left\|\boldsymbol{u}-\boldsymbol{v}_{h}\right\|_{L^{2}\left(\Gamma_{3}\right)^{N}}
\end{aligned}
$$

Using the bounds (6.4)-(6.7) in (6.3) and applying the elementary inequality

$$
a b \leq \delta a^{2}+\frac{1}{4 \delta} b^{2} \quad \forall \delta>0,
$$

we have

$$
\begin{aligned}
\left\|\varepsilon\left(\boldsymbol{u}-\boldsymbol{u}_{h}\right)\right\|_{\mathcal{H}}^{2} & \\
\leq & c\left\{\left\|\boldsymbol{\varepsilon}\left(\boldsymbol{u}-\boldsymbol{v}_{h}\right)\right\|_{\mathcal{H}}^{2}+\left\|\boldsymbol{u}-\boldsymbol{v}_{h}\right\|_{L^{2}\left(\Gamma_{3}\right)^{N}}^{2}+\|F(\varepsilon(\boldsymbol{u}))\|_{\mathcal{H}}\left\|\varepsilon\left(\boldsymbol{u}-\boldsymbol{v}_{h}\right)\right\|_{\mathcal{H}}\right. \\
& \left.+\left(\left\|p_{\nu}\left(u_{\nu}-g\right)\right\|_{L^{2}\left(\Gamma_{3}\right)}+\left\|p_{\tau}\left(u_{\nu}-g\right)\right\|_{L^{2}\left(\Gamma_{3}\right)}\right)\left\|\boldsymbol{u}-\boldsymbol{v}_{h}\right\|_{L^{2}\left(\Gamma_{3}\right)^{N}}\right\}
\end{aligned}
$$

so the inequality (6.2) holds.

The inequality (6.2) is the basis for convergence analysis. Indeed, we see immediately that the finite element method converges under the basic solution regularity $\boldsymbol{u} \in V$, as long as the finite element triangulation is regular and the finite element space $V_{h}$ contains piecewise linear functions. 
We can improve the estimate (6.2) under the regularity assumption $\boldsymbol{\sigma}_{\tau} \in$ $\left(L^{2}\left(\Gamma_{3}\right)\right)^{N}$. In this case, we can perform integration by parts to obtain

$$
R_{2}=\int_{\Gamma_{3}}\left[\boldsymbol{\sigma}_{\tau} \cdot\left(\boldsymbol{v}_{h \tau}-\boldsymbol{u}_{\tau}\right)+p_{\tau}\left(u_{\nu}-g\right)\left(\left|\boldsymbol{v}_{h \tau}\right|-\left|\boldsymbol{u}_{\tau}\right|\right)\right] d a .
$$

Therefore we can use

$$
\left.\left|R_{2}\right| \leq\left(\left\|\boldsymbol{\sigma}_{\tau}\right\|_{L^{2}\left(\Gamma_{3}\right)^{N}}+\left\|p_{\tau}\left(u_{\nu}-g\right)\right\|_{L^{2}\left(\Gamma_{3}\right)}\right)\left\|\boldsymbol{u}_{\tau}-\boldsymbol{v}_{h \tau}\right\|_{L^{2}\left(\Gamma_{3}\right)^{N}}\right)
$$

to replace (6.5). As a result we have the following variant of Theorem 6.1.

TheOREM 6.2. Under the assumptions of Theorem 4.1 with the same value of $L_{0}$, assume additionally $\boldsymbol{\sigma}_{\tau} \in\left(L^{2}\left(\Gamma_{3}\right)\right)^{N}$. Then for some constant $c>0$, we have

$$
\begin{aligned}
& \left\|\boldsymbol{\varepsilon}\left(\boldsymbol{u}-\boldsymbol{u}_{h}\right)\right\|_{\mathcal{H}} \\
& \quad \leq \quad \inf _{\boldsymbol{v}_{h} \in V_{h}}\left\{\left\|\boldsymbol{\varepsilon}\left(\boldsymbol{u}-\boldsymbol{v}_{h}\right)\right\|_{\mathcal{H}}+\left\|\boldsymbol{u}-\boldsymbol{v}_{h}\right\|_{L^{2}\left(\Gamma_{3}\right)^{N}}\right. \\
& \left.\quad+\left(\left\|\boldsymbol{\sigma}_{\tau}\right\|_{L^{2}\left(\Gamma_{3}\right)^{N}}+\left\|p_{\tau}\left(u_{\nu}-g\right)\right\|_{L^{2}\left(\Gamma_{3}\right)}\right)^{1 / 2}\left\|\boldsymbol{u}-\boldsymbol{v}_{h}\right\|_{L^{2}\left(\Gamma_{3}\right)^{N}}^{1 / 2}\right\} .
\end{aligned}
$$

To derive an error estimate, we need to make additional assumptions on the solution regularity. We present a sample result.

Assume

$$
\boldsymbol{u} \in H^{2}(\Omega)^{N},\left.\quad \boldsymbol{u}\right|_{\Gamma_{3}} \in H^{2}\left(\Gamma_{3}\right)^{N} .
$$

We use linear elements for the finite element space $V_{h}$. Let $\Pi_{h} \boldsymbol{u} \in V_{h}$ be the finite element interpolant of the solution $\boldsymbol{u}$. Then from (6.2), we obtain

$$
\begin{aligned}
\left\|\boldsymbol{u}-\boldsymbol{u}_{h}\right\|_{V} \leq & c\left\{\left\|\boldsymbol{u}-\Pi_{h} \boldsymbol{u}\right\|_{V}+\left\|\boldsymbol{u}-\Pi_{h} \boldsymbol{u}\right\|_{L^{2}\left(\Gamma_{3}\right)^{N}}\right. \\
& \left.+\left(\left\|\boldsymbol{\sigma}_{\tau}\right\|_{L^{2}\left(\Gamma_{3}\right)^{N}}+\left\|p_{\tau}\left(u_{\nu}-g\right)\right\|_{L^{2}\left(\Gamma_{3}\right)}\right)^{1 / 2}\left\|\boldsymbol{u}-\Pi_{h} \boldsymbol{u}\right\|_{L^{2}\left(\Gamma_{3}\right)^{N}}^{1 / 2}\right\} .
\end{aligned}
$$

The standard finite element interpolation theory yields (cf. [2])

$$
\left\|\boldsymbol{u}-\Pi_{h} \boldsymbol{u}\right\|_{V} \leq C h|\boldsymbol{u}|_{H^{2}(\Omega)^{N}}, \quad\left\|\boldsymbol{u}-\Pi_{h} \boldsymbol{u}\right\|_{L^{2}\left(\Gamma_{3}\right)^{N}} \leq c h^{2}|\boldsymbol{u}|_{H^{2}\left(\Gamma_{3}\right)^{N}} .
$$

Therefore, under the regularity assumption (6.10), we have the following error estimate:

$$
\begin{aligned}
\left\|\boldsymbol{u}-\boldsymbol{u}_{h}\right\|_{V} \leq & \operatorname{ch}\left(|\boldsymbol{u}|_{H^{2}(\Omega)^{N}}+|\boldsymbol{u}|_{H^{2}\left(\Gamma_{3}\right)^{N}}\right. \\
& \left.+\left(\left\|\boldsymbol{\sigma}_{\tau}\right\|_{L^{2}\left(\Gamma_{3}\right)^{N}}+\left\|p_{\tau}\left(u_{\nu}-g\right)\right\|_{L^{2}\left(\Gamma_{3}\right)}\right)^{1 / 2}|\boldsymbol{u}|_{H^{2}\left(\Gamma_{3}\right)^{N}}^{1 / 2}\right)
\end{aligned}
$$

The finite element system (6.1) can be approximated by a fixed-point iteration method. This follows from a discrete analogue of the proof of Theorem 4.1. Choosing an initial guess $\boldsymbol{u}_{h}^{0} \in V_{h}$, we define a sequence $\left\{\boldsymbol{u}_{h}^{n}\right\} \subset V_{h}$ recursively by

$$
\begin{aligned}
\left(F\left(\varepsilon\left(\boldsymbol{u}_{h}^{n+1}\right)\right), \boldsymbol{\varepsilon}\left(\boldsymbol{v}_{h}\right)-\boldsymbol{\varepsilon}\left(\boldsymbol{u}_{h}^{n+1}\right)\right)_{\mathcal{H}} & +j\left(\boldsymbol{u}_{h}^{n}, \boldsymbol{v}_{h}\right)-j\left(\boldsymbol{u}_{h}^{n}, \boldsymbol{u}_{h}^{n+1}\right) \\
& \geq\left(\boldsymbol{f}, \boldsymbol{v}_{h}-\boldsymbol{u}_{h}^{n+1}\right)_{V} \quad \forall \boldsymbol{v}_{h} \in V_{h} .
\end{aligned}
$$


We have the following convergence result.

THEOREM 6.3. Under the assumptions of Theorem 4.1 with the same value of $L_{0}$, the iteration method (6.11) converges:

$$
\left\|\boldsymbol{u}_{h}^{n}-\boldsymbol{u}_{h}\right\|_{V} \rightarrow 0 \quad \text { as } n \rightarrow \infty \text {. }
$$

Furthermore, for some constant $0<\kappa<1$, we have the estimate

$$
\left\|\boldsymbol{u}_{h}^{n}-\boldsymbol{u}_{h}\right\|_{V} \leq c \kappa^{n} \text {. }
$$

Proof. We take $\boldsymbol{v}_{h}=\boldsymbol{u}_{h}^{n+1}$ in (6.1),

$$
\begin{array}{r}
\left(F\left(\varepsilon\left(\boldsymbol{u}_{h}\right)\right), \boldsymbol{\varepsilon}\left(\boldsymbol{u}_{h}^{n+1}\right)-\boldsymbol{\varepsilon}\left(\boldsymbol{u}_{h}\right)\right)_{\mathcal{H}}+j\left(\boldsymbol{u}_{h}, \boldsymbol{u}_{h}^{n+1}\right)-j\left(\boldsymbol{u}_{h}, \boldsymbol{u}_{h}\right) \\
\geq\left(\boldsymbol{f}, \boldsymbol{u}_{h}^{n+1}-\boldsymbol{u}_{h}\right)_{V},
\end{array}
$$

and take $\boldsymbol{v}_{h}=\boldsymbol{u}_{h}$ in (6.11),

$$
\begin{aligned}
\left(F\left(\varepsilon\left(\boldsymbol{u}_{h}^{n+1}\right)\right), \boldsymbol{\varepsilon}\left(\boldsymbol{u}_{h}\right)-\boldsymbol{\varepsilon}\left(\boldsymbol{u}_{h}^{n+1}\right)\right)_{\mathcal{H}}+j\left(\boldsymbol{u}_{h}^{n}, \boldsymbol{u}_{h}\right) & -j\left(\boldsymbol{u}_{h}^{n}, \boldsymbol{u}_{h}^{n+1}\right) \\
& \geq\left(\boldsymbol{f}, \boldsymbol{u}_{h}-\boldsymbol{u}_{h}^{n+1}\right)_{V} .
\end{aligned}
$$

Adding (6.13) and (6.14), we obtain

$$
\begin{aligned}
\left(F\left(\varepsilon\left(\boldsymbol{u}_{h}\right)\right)-F\left(\varepsilon\left(\boldsymbol{u}_{h}^{n+1}\right)\right), \varepsilon\left(\boldsymbol{u}_{h}\right)-\varepsilon\left(\boldsymbol{u}_{h}^{n+1}\right)\right) \leq & j\left(\boldsymbol{u}_{h}, \boldsymbol{u}_{h}^{n+1}\right)-j\left(\boldsymbol{u}_{h}, \boldsymbol{u}_{h}\right) \\
& +j\left(\boldsymbol{u}_{h}^{n}, \boldsymbol{u}_{h}\right)-j\left(\boldsymbol{u}_{h}^{n}, \boldsymbol{u}_{h}^{n+1}\right) .
\end{aligned}
$$

Then as in the proof of Lemma 4.4, we can derive the estimate

$$
\begin{aligned}
\left\|\varepsilon\left(\boldsymbol{u}_{h}-\boldsymbol{u}_{h}^{n+1}\right)\right\|_{\mathcal{H}} & \leq \frac{c_{0}^{2}}{m}\left(L_{\nu}+L_{\tau}\right)\left\|\varepsilon\left(\boldsymbol{u}_{h}-\boldsymbol{u}_{h}^{n}\right)\right\|_{\mathcal{H}} \\
& =\frac{L_{\nu}+L_{\tau}}{L_{0}}\left\|\varepsilon\left(\boldsymbol{u}_{h}-\boldsymbol{u}_{h}^{n}\right)\right\|_{\mathcal{H}} .
\end{aligned}
$$

Under the stated assumption, $\kappa \equiv\left(L_{\nu}+L_{\tau}\right) / L_{0}<1$, and we have the estimate (6.12).

\section{References}

[1] K. T. Andrews, A. Klarbring, M. Shillor and S. Wright, A dynamic thermoviscoelastic body in frictional contact with a rigid obstacle, Eur. J. Appl. Math., to appear.

[2] P. G. Ciarlet, The Finite Element Method for Elliptic Problems, North-Holland, Amsterdam, 1978.

[3] M. Cocu, Existence of solutions of Signorini problems with friction, Int. J. Engrg. Sci. 22 (1984), 567-581.

[4] -, Unilateral contact problems with friction for an elastoviscoplastic material with internal state variable, in: Proc. Contact Mechanics Int. Symp., A. Curnier (ed.), PPUR, 1992, 207-216.

[5] G. Duvaut, Loi de frottement non locale, J. Méc. Théor. Appl., Special issue 1982, $73-78$. 
[6] G. Duvaut and J. L. Lions, Inequalities in Mechanics and Physics, Springer, Berlin, 1976.

[7] W. Han, On the numerical approximation of a frictional contact problem with normal compliance, Numer. Funct. Anal. Optim. 17 (1996), 307-321.

[8] I. R. Ionescu and M. Sofonea, Functional and Numerical Methods in Viscoplasticity, Oxford Univ. Press, Oxford, 1993.

[9] N. Kikuchi and J. T. Oden, Contact Problems in Elasticity: A Study of Variational Inequalities and Finite Element Methods, SIAM, Philadelphia, 1988.

[10] A. Klarbring, A. Mikelič and M. Shillor, Frictional contact problems with normal compliance, Int. J. Engrg. Sci. 26 (1988), 811-832.

[11] J. A. C. Martins and J. T. Oden, Existence and uniqueness results for dynamic contact problems with nonlinear normal and friction interface laws, Nonlinear Anal. 11 (1987), 407-428.

[12] J. Nečas and I. Hlaváček, Mathematical Theory of Elastic and Elastoplastic Bodies: An Introduction, Elsevier, Amsterdam, 1981.

[13] P. D. Panagiotopoulos, Inequality Problems in Mechanics and Applications, Birkhäuser, Basel, 1985.

[14] M. Rochdi, M. Shillor and M. Sofonea, A quasistatic viscoelastic contact problem with normal compliance and friction, J. Elasticity 51 (1998), 105-126.

[15] M. Shillor and M. Sofonea, A quasistatic viscoelastic contact problem with friction, Int. J. Engrg. Sci., to appear.

[16] N. Strömberg, Continuum Thermodynamics of Contact, Friction and Wear, Ph.D. Thesis, Linköping University, 1995.

[17] N. Strömberg, L. Johansson and A. Klarbring, Derivation and analysis of a generalized standard model for contact friction and wear, Int. J. Solids Structures 33 (1996), 1817-1836.

[18] E. Zeidler, Nonlinear Functional Analysis and its Applications. IV: Applications to Mathematical Physics, Springer, New York, 1988.

Weimin Han

Department of Mathematics

University of Iowa

Iowa City, IA 52242, USA

E-mail: whan@math.uiowa.edu
Mircea Sofonea Laboratoire de Théorie des Systèmes Université de Perpignan 52 Avenue de Villeneuve 66860 Perpignan, France E-mail: sofonea@univ-perp.fr 